MRI-PHY/96/03

\title{
Fluctuation Effects And Order Parameter Symmetry In The Cuprate Superconductors
}

\author{
Biplab Chattopadhyay, D. M. Gaitonde and A. Taraphder \\ Mehta Research Institute \\ 10 Kasturba Gandhi Marg, Allahabad 211002, India.
}

\begin{abstract}
Effect of phase fluctuations on superconducting states with anisotropic order parameters is studied in a BCS like lattice model of cuprate superconductors. The degradation of the mean field transition temperature due to phase fluctuations is estimated within a Kosterlitz-Thouless scenario. Values of the interaction parameters for optimal doping, corresponding to a stable superconducting state of $S_{x y}$ symmetry, which fit the nodal structure of the superconducting order parameter in the Bi2212 compound, are obtained. The angular position of the node is found to be insensitive to the dopant concentration.
\end{abstract}

PACS numbers: 74.20.Mn, 74.20.Fg, 74.72.-h 
The symmetry of the order parameter (OP) in high temperature superconductors continues to be a matter of much controversy. A series of experiments [1-6] have indicated that the OP in high temperature superconductors is highly anisotropic [7]. However, these experiments have conflicting features [8,9] which have not yet made it possible to determine the symmetry of the OP in an unambiguous way. Recent angle resolved photoemission spectroscopy (ARPES) experiments [5, 6] have been able to shed new light on the symmetry of the OP, owing to their high resolution $(\sim 10 \mathrm{meV})$ compared to the size of the superconducting gaps in the cuprates $(\sim 50 \mathrm{meV})$. For high quality Bi2212 single-crystal samples, the gap on the Fermi surface (FS) vanishes at two points symmetrically displaced from the $45^{\circ}$ direction in the Brillouin zone by about 10 degrees [6] at the optimum dopant concentration $(\delta=0.17)$ which has the highest Tc. Such a nodal structure is inconsistent with the $d_{x^{2}-y^{2}}$ symmetry, which has only one node per quadrant along the $45^{\circ}$ direction. Also, a small admixture of an isotropic S-wave component to the $d_{x^{2}-y^{2}}$ OP does not increase the number of nodes but merely displaces the node away from the $45^{\circ}$ direction.

Norman and coworkers have, in a recent set of papers [10], made a strong case for an OP whose symmetry is predominantly $S_{x y}$. They considered a phenomenological model of electrons on a square lattice with an on-site repulsion $\left(V_{0}\right)$ and attractive nearest neighbour $\left(V_{1}\right)$ as well as next nearest neighbour $\left(V_{2}\right)$ interactions. The Hamiltonian of the system is given by $\mathrm{H}=\mathrm{H}_{0}+\mathrm{H}_{1}$ where

$$
H_{0}=\sum_{k, \sigma}\left(\epsilon_{\vec{k}}-\epsilon_{F}\right) c_{\vec{k}, \sigma}^{\dagger} c_{\vec{k}, \sigma}
$$

and

$$
H_{1}=V_{0} \sum_{i} \hat{n}_{i, \uparrow} \hat{n}_{i, \downarrow}+V_{1} \sum_{i, \sigma, \sigma^{\prime}} \sum_{\delta_{n n}} \hat{n}_{i, \sigma} \hat{n}_{i+\delta_{n n}, \sigma^{\prime}}+V_{2} \sum_{i, \sigma, \sigma^{\prime}} \sum_{\delta_{n n n}} \hat{n}_{i, \sigma} \hat{n}_{i+\delta_{n n n}, \sigma^{\prime}}
$$

The band dispersion $\epsilon_{\vec{k}}$ was chosen to fit the normal state quasiparticle dispersion obtained from the ARPES data on Bi2212 single crystals [10]. A six parameter tight binding Hamiltonian with $\left[t_{0}, \ldots, t_{5}\right]=[0.131,-0.149,0.041,-0.013,-0.014,0.013]$ (in eV) was found to reproduce the observed quasiparticle dispersion. Here $t_{0}$ is the orbital energy, $t_{1}$ nearest neighbour ( $\left.n n\right), t_{2}$ next nearest neighbour (nnn) etc. hopping matrix elements.

A mean field analysis of the spin-singlet pairing instabilities of the above model [10] showed that the strongest instabilities correspond to OP's with $S_{x y}$ and $d_{x^{2}-y^{2}}$ symmetries because they best exploit the large single particle density of states (DOS) just below the FS; their relative stability is determined by the ratio of $V_{1}$ and $V_{2}$. A consistent fit to the ARPES data could be obtained in a range of values of $V_{1}$ and $V_{2}$ while $V_{0}$ was constrained to be small.

However, the above calculations overlook the strong fluctuations that exist in the cuprates owing to their quasi two dimensional nature. In this letter, we study the phase diagram of the Hamiltonian in Eq.(1) with the incorporation of the effects of fluctuations 
in the phase of the OP. We calculate the superfluid phase stiffness or helicity modulus $\left(\rho_{s}\right)$ below the mean field transition temperature $\left(T_{c}^{M F}\right)$, and use it in conjunction with the Kosterlitz-Thouless (KT) relation $\rho_{s}\left(T_{c}^{K T}\right)=\frac{2}{\pi} k_{B} T_{c}^{K T}$ to determine the reduced transition temperature $\left(T_{c}^{K T}\right)$. The interplanar Josephson coupling, that exists in the cuprates, does not lead to a strict KT like scenario as is being assumed here (except in thin films). However, the magnitude of $T_{c}^{K T}$ is determined by $\rho_{s}$ and can therefore be expected to yield a good estimate of $T_{c}$ degradation due to phase fluctuations. With this improved bound on $T_{c}$, we determine the relative stability of superconducting OP's with $\mathrm{B}_{1}\left(d_{x^{2}-y^{2}}\right), \mathrm{B}_{2}\left(d_{x y}\right)$ and $\mathrm{A}_{1}$ (a 3 dimensional representation comprising $S, S^{\star}$ and $S_{x y}$ ) symmetries. We also study the nodal structure of the gap function on the FS.

We find that the region of stability of the $\mathrm{A}_{1}\left(S_{x y}\right)$ OP with respect to $d_{x^{2}-y^{2}}$ and $d_{x y}$, is reduced compared to the mean field result. An OP which is predominantly $S_{x y}$ with very small isotropic S-wave $(S)$ and extended S-wave $\left(S^{\star}\right)$ components correctly reproduces the gap structure predicted from ARPES measurements. The best fit, corresponding to a $T_{c}^{K T} \sim 100 \mathrm{~K}$ and a stable $\mathrm{A}_{1}$ phase with a nodal structure consistent with ARPES measurements, occurs for $V_{0} \leq 15 \mathrm{meV}, V_{1}$ in the range $30-45 \mathrm{meV}$ and $V_{2}$ around $70-75 \mathrm{meV}$. For these parameters we find the variation of the transition temperature $\left(T_{c}^{K T}\right)$ to be qualitatively correct while the angular positions of the nodes on the FS are nearly independent of $\delta$.

Standard mean field factorization of the Hamiltonian (Eq.(1)) gives the gap equation

$$
\Delta_{\vec{k}}=\frac{1}{N} \sum_{\vec{k}^{\prime}} V\left(\vec{k}-\vec{k}^{\prime}\right) \frac{\Delta_{\vec{k}^{\prime}}}{2 E_{\vec{k}^{\prime}}} \tanh \left(\frac{\beta E_{\vec{k}^{\prime}}}{2}\right)
$$

where the quasiparticle energy is $E_{\vec{k}}=\sqrt{\left(\epsilon_{\vec{k}}-\epsilon_{F}\right)^{2}+\left|\Delta_{\vec{k}}\right|^{2}}$ and $\Delta_{\vec{k}}$ is the BCS gap function. The pairing interaction, $V(q)=V_{0}+4 V_{1}\left(\cos q_{x}+\cos q_{y}\right)+8 V_{2} \cos q_{x} \cos q_{y}$, is separable and can be written as $V\left(\vec{k}-\vec{k}^{\prime}\right)=\sum_{i=0}^{4} \tilde{V}_{i} \eta_{i}(\vec{k}) \eta_{i}\left(\vec{k}^{\prime}\right)$ where $\eta_{0}(\vec{k})=1, \eta_{1}(\vec{k})=$ $\frac{1}{2}\left(\cos k_{x}+\cos k_{y}\right), \eta_{2}(\vec{k})=\frac{1}{2}\left(\cos k_{x}-\cos k_{y}\right), \eta_{3}(\vec{k})=\cos k_{x} \cos k_{y}, \eta_{4}(\vec{k})=\sin k_{x} \sin k_{y}$, corresponding to $\left[S, S^{\star}, d_{x^{2}-y^{2}}, S_{x y}, d_{x y}\right]$ symmetries, and $\left(\tilde{V}_{0} \ldots \tilde{V}_{4}\right)=\left(V_{0}, 8 V_{1}, 8 V_{1}, 8 V_{2}\right.$, $\left.8 V_{2}\right)$. We have ignored terms corresponding to triplet pairing. It is easy to see that upon writing the gap functions as $\Delta_{\vec{k}}=\sum_{i} \eta_{i}(\vec{k}) \Delta_{i}$, the linearized gap equation (whose non-vanishing solution first appears at $T_{c}^{M F}$ ) factorizes into two independent equations for $\Delta_{2}$ and $\Delta_{4}\left(\mathrm{~B}_{1}\right.$ and $\mathrm{B}_{2}$ representations) and three coupled linear equations involving $\Delta_{0}, \Delta_{1}, \Delta_{3}\left(\mathrm{~A}_{1}\right.$ representation). Since $\Delta_{3}$ is the largest component within the $\mathrm{A}_{1}$ representation, the OP symmetry is predominantly $S_{x y}$. We ignore the possibility of further transitions at lower temperatures.

We now proceed to the calculation of the $\rho_{s}$ and $T_{c}^{K T}$. We first solve the gap equations below $T_{c}^{M F}$, choosing the gap function $\Delta_{\vec{k}}=\sum_{i \in \mathcal{R}} \Delta_{i} \eta_{i}(\vec{k})$. As an illustration, we plot the gap functions $\Delta_{0}, \Delta_{1}, \Delta_{3}\left(\mathrm{~A}_{1}\right)$ and $\Delta_{2}, \Delta_{4}\left(\mathrm{~B}_{1}\right.$ and $\mathrm{B}_{2}$ respectively) in the inset of Fig.1, as a function of temperature for optimal parameters (see below). To calculate $\rho_{s}$, we introduce a transverse vector potential and choose the gauge $A_{y}=0$. Then, the 
hopping matrix elements in $H_{0}$ (Eq.(1a)) acquire an additional phase factor according to the Peierls substitution $t_{i j} \rightarrow t_{i j} \exp \left[\frac{i e}{\hbar c} \int_{\vec{R}_{j}}^{\vec{R}_{i}} \vec{A} \cdot \overrightarrow{d l}\right]$.

The electron current operator $\hat{j}_{x}\left(\vec{R}_{i}\right)$ can be found (to linear order in $A_{x}$ ) by differentiating $H_{0}$ with respect to $A_{x}\left(\vec{R}_{i}\right)$ and is of the form

$$
\hat{j}_{x}\left(\vec{R}_{i}\right)=-c \frac{\partial H_{0}}{\partial A_{x}\left(\vec{R}_{i}\right)}=\hat{j}_{x}^{\text {para }}\left(\vec{R}_{i}\right)+\hat{j}_{x}^{\text {dia }}\left(\vec{R}_{i}\right)
$$

The first term on the RHS of Eq.(3) is of zeroth order in $A_{x}$ and is the electron velocity operator. It represents the paramagnetic response of the quasi-particles. The second term is of linear order in $A_{x}$ and its physical origin is related to the Meissner screening of the condensate. The average diamagnetic current is obtained to be

$$
j_{x}^{d i a}(\vec{q})=-\frac{e^{2}}{\hbar^{2} c} \frac{1}{N} \sum_{\vec{k}, \sigma}\left\langle c_{\vec{k}, \sigma}^{\dagger} c_{\vec{k}, \sigma}\right\rangle \frac{\partial^{2} \epsilon_{\vec{k}}}{\partial k_{x}^{2}} A_{x}(\vec{q})
$$

where the angular brackets correspond to an average in the mean field superconducting state. From Eq.(4) we see that the diamagnetic contribution to the phase stiffness $\left(\rho_{s}^{d i a}\right)$ is proportional to $\frac{1}{N} \sum_{\vec{k}, \sigma}\left\langle c_{\vec{k}, \sigma}^{\dagger} c_{\vec{k}, \sigma}\right\rangle \frac{\partial^{2} \epsilon_{\vec{k}}}{\partial k_{x}^{2}}$, the mean electronic kinetic energy along the $\mathrm{x}$-direction [11]. Unlike models in which the electron dispersion is parabolic, $\rho_{s}^{\text {dia }}$ for a lattice superconductor is not proportional to the average electronic density. Thus the use [12] of the continuum expression $\rho_{s}^{d i a} \propto-\sum_{\vec{k}, \sigma}\left(\frac{\partial \epsilon_{\vec{k}}}{\partial k_{x}}\right)^{2} \frac{\partial f\left(\epsilon_{\vec{k}}-\epsilon_{F}\right)}{\partial \epsilon_{\vec{k}}}$ (where $f$ is the Fermi function) is incorrect and could leave out important physics. be

From linear response theory, the long wavelength paramagnetic current is found to

$$
j_{x}^{\text {para }}(\vec{q})=-\frac{1}{c}\left[\lim _{\vec{q} \rightarrow 0} \lim _{\omega \rightarrow 0} K^{x x}(\vec{q}, \omega)\right] A_{x}(\vec{q})
$$

where $K^{x x}(\vec{q}, \omega)=-\imath \int d t \theta(t) e^{\imath \omega t}\left\langle\left[j_{x}^{\text {para }}(\vec{q}, t), j_{x}^{\text {para }}(-\vec{q}, 0)\right]\right\rangle$. We work in a transverse gauge and vertex corrections required to get a gauge invariant current have been ignored. The correlation function in Eq.(5a) is easily evaluated and found to be

$$
\lim _{\vec{q} \rightarrow 0} \lim _{\omega \rightarrow 0} K^{x x}(\vec{q}, \omega)=\frac{e^{2}}{\hbar^{2}} \frac{1}{N} \sum_{\vec{k}}\left(\frac{\partial \epsilon_{\vec{k}}}{\partial k_{x}}\right)^{2} \frac{\partial f\left(E_{\vec{k}}\right)}{\partial E_{\vec{k}}}
$$

From Eqs (4) and (5) we obtain the superfluid phase stiffness

$$
\rho_{s}(T)=\frac{1}{2 N} \sum_{\vec{k}}\left[\left(\frac{\partial \epsilon_{\vec{k}}}{\partial k_{x}}\right)^{2} \frac{\partial f\left(E_{\vec{k}}\right)}{\partial E_{\vec{k}}}+\frac{1}{2} \frac{\partial^{2} \epsilon_{\vec{k}}}{\partial k_{x}^{2}}\left\{1-\frac{\epsilon_{\vec{k}}-\epsilon_{F}}{E_{\vec{k}}} \tanh \left(\frac{\beta E_{\vec{k}}}{2}\right)\right\}\right] .
$$

In Fig. 1 we show $\rho_{s}$ as a function of temperature for various OP symmetries. The results indicate that the temperature dependence of $\rho_{s}$ does not show any major qualitative change for the various types of OP's considered. Thus the fluctuation corrected phase boundaries are not expected to be very different from the mean field case. 
The KT transition temperature $\left(T_{c}^{K T}\right)$ is found by solving

$$
\rho_{s}\left(T_{c}^{K T}\right)=\frac{2}{\pi} T_{c}^{K T}
$$

We use the expression for $\rho_{s}$ (Eq.(6) in Eq.(7)), assuming mean field values of the OP, and obtain $T_{c}^{K T}$. This procedure was employed earlier by Denteneer et. al. [13 in a study of the 2-d attractive Hubbard model and gives a qualitatively correct picture even in the limit of strong coupling where $T_{c}^{K T}$ is inversely proportional to the coupling. Since we neglect the renormalization of the OP (and hence $\rho_{s}$ ), due to the presence of vortex like fluctuations, the discontinous drop in $\rho_{s}$, characteristic of KT transitions, is not obtainable here. Hence our results give an upper bound on the true KT transition temperature. Another possible difficulty is that the expression in Eq.(7) has been derived for isotropic S-wave superconductors. However, even in the anisotropic case, the asymptotic form of the vortex-vortex interaction (at high vortex density) is logarithmic [14] and so corrections to Eq.(7) are likely to be small. A comparison of $T_{c}^{K T}$ thus obtained for different OP's enable us to determine the phase diagram of the model Hamiltonian.

We now discuss our results. For the sake of clarity we present the phase diagrams in the $V_{0}-V_{2}$ and $V_{1}-V_{2}$ planes only. In Fig.2 we have drawn the phase diagram in the $V_{0}-V_{2}$ plane (we set $V_{1}=0$ ) for several values of $\delta$. For comparison, we also include the mean field phase boundaries [10]. The dopant concentration $(\delta)$ indicates the deviation from half-filling. It is clear from Fig.2 that fluctuation effects are negligible at weak coupling $\left(\left|V_{2}\right|<50 \mathrm{meV}\right)$. To obtain a stable $S_{x y}\left(\mathrm{~A}_{1}\right)$ solution at low filling, $V_{0}$ is required to be rather small $\left(V_{0}<0.4 \mathrm{eV}\right.$ at $\left.\delta=0.17\right)$, even smaller than the value indicated by the mean field analysis and larger values of $\left|V_{2}\right| / V_{0}$ are required to stabilize the $S_{x y}$ state. At high values of $\delta$, the $\mathrm{A}_{1}$ phase is stable for much larger values of $V_{0}$ (a few $\mathrm{eV}$ ) provided $\left|V_{2}\right| \leq 90 \mathrm{meV}$. This is understandable as the onsite repulsion, which disfavours the $\mathrm{A}_{1}$ state, has the largest effect close to half filling and becomes relatively ineffective at low filling.

In Fig.3 we plot the phase boundary separating the $d_{x^{2}-y^{2}}$ and $\mathrm{A}_{1}$ superconducting states in the $V_{1}-V_{2}$ plane (setting $V_{0}=0$ ). Fluctuation effects are once again seen to be negligible at weak coupling but lead to a larger critical value of $V_{2} / V_{1}$ (to stabilize the $S_{x y}$ state) at intermediate and strong couplings. Both the mean field and KT phase boundaries are insensitive to $\delta$. This is not surprising as both the $d_{x^{2}-y^{2}}$ and $S_{x y}$ solutions have a very similar pairing density of states [10] and thus the phase boundary is determined almost entirely by the interaction strength.

We have found the values of interaction parameters at $\delta=0.17$ (optimal doping) that produce a stable superconducting state with the $\mathrm{A}_{1}$ symmetry and a transition temperature $T_{c}^{K T} \sim 100 \mathrm{~K}$ whose $\mathrm{OP}$ has the observed nodal structure. We find that the on-site repulsion $V_{0}$ is constrained to be small $(\leq 15 \mathrm{meV}), V_{2}$ lies in the range $70<-V_{2}<75 \mathrm{meV}$ whereas $V_{1}$ can be chosen in a relatively broader range $30<$ $-V_{1}<45 \mathrm{meV}$. In Fig.4 we have plotted the angular dependence of $\left|\Delta_{\mathbf{k}}(T=0)\right|$ on the FS $(\phi$ measures the angular deviation from the line passing through the $(0, \pi)$ and 
$(\pi, \pi)$ points), for typical values of $V_{0}, V_{1}$ and $V_{2}$, lying within this range. A puzzling feature of these results, which does not have any apparent microscopic justification, is the extremely small value of $V_{0}$, whereas the actual bare $\mathrm{Cu}$ onsite repulsion is known to be about a few $\mathrm{eV}$.

We have studied the variation of $T_{c}^{K T}$ with $\delta$ for the optimal parameters determined above. We find that $T_{c}^{K T}$ has the correct qualitative behaviour (see inset of Fig.5) with the highest $T_{c}^{K T}$ being achieved for $\delta \approx 0.25$. This is not surprising as the pairing density of states (for a superconducting instability in the $\mathrm{A}_{1}$ state) at the Fermi energy is peaked close to this value of $\delta$ [10]. We have also found the nodal structure of the $\mathrm{OP}$ as a function of $\delta$. The magnitude of the gap function on the FS away from the nodes is strongly dependent on $\delta$ (reflecting the variation in $T_{c}^{K T}$ ). However the angular positions of the nodes on the FS are insensitive to $\delta$ (see Fig.5 and its inset) and stay close to their positions for $\delta=0.17$.

In conclusion, we have studied the competition between superconducting OP's with different symmetries within a phenomenological BCS model and obtained a phase diagram which incorporates the effects of fluctuations. Our phase diagram, together with the magnitude of the transition temperature and the nodal structure of the OP, enable us to identify the best choice of parameters appropriate to the optimally doped Bi2212 compound. We find that the transition temperature has the correct doping dependence and the angular positions of the nodes of the gap function on the FS are nearly independent of $\delta$ for the best parameters. 


\section{References}

[1] W. N. Hardy et. al., Phys. Rev. Lett. 70, 3999 (1993); D. H. Wu et. al., Phys. Rev. Lett. 70, 85 (1993).

[2] C. C. Tsuei et. al., Phys. Rev. Lett. 73, 593 (1994); J. R. Kirtley et. al., Nature (London) 373, 225 (1995).

[3] D. A. Wollman et. al., Phys. Rev. Lett 71, 2134 (1993); ibid 74, 797 (1995); D.

A. Brawner and H. R. Ott, Phys. Rev. B 50, 6530 (1994).

[4] P. Chaudhari and S. -Y. Lin, Phys. Rev. Lett 72, 1084 (1994).

[5] Z. -X. Shen et. al., Phys. Rev. Lett. 70, 1553 (1993).

[6] H. Ding et. al., Phys. Rev. Lett. 74, 2784 (1995).

[7] M. Sigrist and K. Ueda, Rev. Mod. Phys. 63, 239 (1991); J. Annet et. al., Preprint cond-mat/9601060.

[8] D. J. Scalapino, Physics Reports 250, 392 (1995).

[9] S. Chakravarty, A. Sudbø and P. W. Anderson, Science 261, 3999 (1993).

[10] R. Fehrenbacher and M. R. Norman, Phys. Rev. Lett. 74, 3884 (1995); M. R. Norman et. al., Phys. Rev. B 52, 615 (1995).

[11] D. J. Scalapino, S. R. White and S. C. Zhang, Phys. Rev. Lett. 68, 2830 (1992).

[12] C. O’Donnovan and J. P. Carbotte. Physica C252, 87 (1995).

[13] P. J. H. Denteneer et. al., Europhys. Lett. 16, 5 (1991).

[14] Y. Ren et. al., Phys. Rev. Lett. 74, 3680 (1995). 


\section{FIGURE CAPTIONS}

Fig.1. $\rho_{s}$ as a function of temperature at $\delta=0.17, V_{0}=15 \mathrm{meV}, V_{1}=-40 \mathrm{meV}$ and $V_{2}=-75 \mathrm{meV}$ for the $\mathrm{A}_{1}, \mathrm{~B}_{1}$ and $\mathrm{B}_{2}$ symmetries. The intersection of the straight line with $\rho_{s}$ gives $T_{c}^{K T}$. Inset shows the variation of the mean field OP's with temperature.

Fig.2. The mean field (dashed lines) and KT (solid lines) phase boundaries indicating the regions of stability of the $\mathrm{A}_{1}$ and $\mathrm{d}_{x y}$ phases are shown in the $\left(V_{0},-V_{2}\right)$ plane for various $\delta$. The inset shows the KT phase diagram for $\delta=0.17$ on an expanded scale.

Fig.3. The mean field and KT phase boundaries indicating the regions of stability of the $\mathrm{A}_{1}$ and $\mathrm{d}_{x^{2}-y^{2}}$ phases are shown in the $\left(-V_{2},-V_{1}\right)$ plane for various $\delta$. The inset shows the KT phase diagram for $\delta=0.17$ on an expanded scale.

Fig.4. The angular dependence of the gap function $\left|\Delta_{\mathbf{k}}(T=0)\right|$ on the Fermi surface is shown for $\delta=0.17$ and various values of interaction parameters in the optimal range. The angles are measured with respect to the $(\pi, \pi)$ point as in ref. [10].

Fig.5. $\left|\Delta_{\mathbf{k}}(T=0)\right|$ on the Fermi surface with $V_{0}=15 \mathrm{meV}, V_{1}=-40 \mathrm{meV}$ and $V_{2}=-74 \mathrm{meV}$ (optimal parameters) for various $\delta$. Curves 1-5 correspond to $\delta=$ $0.10,0.13,0.17,0.21$ and 0.26 respectively. Inset shows the angular position $(\alpha)$ of the node on the Fermi surface (measured from the $45^{\circ}$ direction) and $T_{c}^{K T}$ (in ${ }^{\circ} \mathrm{K}$ ) as a function of $\delta$ for optimal parameters. 